\title{
The Classical Limit for Quantum Mechanical Correlation Functions
}

\author{
Klaus Hepp \\ Physics Department, ETH, Zürich, Schweiz
}

Received November 5, 1973

\begin{abstract}
For quantum systems of finitely many particles as well as for boson quantum field theories, the classical limit of the expectation values of products of Weyl operators, translated in time by the quantum mechanical Hamiltonian and taken in coherent states centered in $x$ - and $p$-space around $\hbar^{-1 / 2}$ (coordinates of a point in classical phase space) are shown to become the exponentials of coordinate functions of the classical orbit in phase space. In the same sense, $\hbar^{-1 / 2}$ [(quantum operator) $(t)-$ (classical function) $\left.(t)\right]$ converges to the solution of the linear quantum mechanical system, which is obtained by linearizing the non-linear Heisenberg equations of motion around the classical orbit.
\end{abstract}

\section{§ 1. Introduction}

Consider the canonical system with the real Hamilton function

$$
\mathscr{H}(\pi, \xi)=\pi^{2} / 2 m+V(\xi)
$$

in the $2 f$-dimensional phase space $\mathbb{R}^{2 f} \ni(\pi, \xi)$. If $\operatorname{grad} V=\nabla V$ is Lipschitz around $\xi$, then the canonical equations

$$
m \dot{\xi}(t)=\pi(t), \quad \dot{\pi}(t)=-\operatorname{grad} V(\xi(t))
$$

have a unique solution $(\xi(\alpha, t), \pi(\alpha, t))$ for times $|t|<T(\alpha)$ (possibly $0<T(\alpha) \leqq \infty)$ with the initial data

$$
\xi(\alpha, 0)=\xi, \quad \pi(\alpha, 0)=\pi, \quad \alpha=(\xi+i \pi) / \sqrt{2} .
$$

While the classical equations (1.2) have locally unique but globally possibly nonexistent solutions (escape to infinity in finite times or collisions in the $N$-body problem), the corresponding quantum mechanical problem

$$
i \hbar \frac{\partial \psi}{\partial t}(x, t)=-\frac{\hbar^{2}}{2 m} \Delta \psi(x, t)+V(x) \psi(x, t)
$$

in $L^{2}\left(\mathbb{R}^{f}\right)$ has always global solutions, if $p_{\hbar}^{2} / 2 m$ and $V_{\hbar}$ have a common dense domain $\mathscr{D}$ and if $\psi=\psi(\cdot, 0) \in \mathscr{D}$, by taking any selfadjoint exten$\operatorname{sion} H_{\hbar}$ of the real and symmetric operator $p_{\hbar}^{2} / 2 m+V_{\hbar}, U_{\hbar}(t)=\exp \left(-i H_{\hbar} t / \hbar\right)$ 
and $\psi_{t}=U_{\hbar}(t) \psi$. However, these global solutions are not unique, if $p_{\hbar}^{2} / 2 m+V_{\hbar}$ is not essentially self-adjoint on $\mathscr{D}$.

The discussion of the connection between (1.2) and (1.4) is as old as quantum mechanics (see e.g. [1-3]). The WKB method relates an asymptotic expansion of solutions of (1.4) for $\hbar \rightarrow 0$ to solutions of the Hamilton Jacobi equations for (1.2) [4]. For more than one degree of freedom, the mathematical difficulties of this approach are considerable [5]. The Feynman integral approach [6] is very suggestive, but also difficult in rigorous mathematical terms [7]. The simplest connection between quantum and classical mechanics, however, goes back to Ehrenfest [8]: For every $\psi \in \mathscr{D}$ and $V$ sufficiently regular,

$$
\begin{aligned}
& \frac{d}{d t}\left(\psi_{t}, q_{\hbar} \psi_{t}\right)=\left(\psi_{t}, p_{\hbar} \psi_{t}\right) / m \\
& \frac{d}{d t}\left(\psi_{t}, p_{\hbar} \psi_{t}\right)=-\left(\psi_{t}, \nabla V_{h} \psi_{t}\right) .
\end{aligned}
$$

However (1.5) does not define a solution of (1.2) since $\left(\psi_{t}, \nabla V_{\hbar} \psi_{t}\right)$ $\neq \nabla V\left(\left(\psi_{t}, q_{\hbar} \psi_{t}\right)\right)$, unless $\nabla V$ is linear, and even if the error is small for some $t$, it need not be controllable for all $t$, if $h>0$.

It is a folk-theorem (see $[9,36]$ ) that $(1.5)$ establishes a rigorous transition to (1.2), when $\hbar \rightarrow 0$ in minimal uncertainty states for $p_{\hbar}$ and $q_{h}$, i.e. in coherent states [10] centered around large mean values $\hbar^{-1 / 2} \pi$, $\hbar^{-1 / 2} \xi$. This becomes apparent in the following symmetric representation of the CCR:

$$
p_{\hbar}=\sqrt{\hbar} p, \quad q_{\hbar}=\sqrt{\hbar} q,
$$

where $p=-i d / d x, q=x$ and $a=(q+i p) / \sqrt{2}$ are $\hbar$-independent. Let $\alpha \in \mathbb{C}$ and

$$
U(\alpha)=\exp \left(\alpha a^{*}-\alpha^{*} a\right)=\exp i(\pi q-\xi p) .
$$

Because of $U(\alpha) a U(\alpha)^{*}=a-\alpha$, one has in the coherent state $|\alpha\rangle=U(\alpha)|0\rangle$ (where $a|0\rangle=0$ ) for an arbitrary monomial in the $p$ 's and $q$ 's:

$$
\left\langle\hbar^{-1 / 2} \alpha\left|\left(q-\hbar^{-1 / 2} \xi\right) \ldots\left(p-\hbar^{-1 / 2} \pi\right)\right| \hbar^{-1 / 2} \alpha\right\rangle=\langle 0|q \ldots p| 0\rangle,
$$

and hence

$$
\lim _{\hbar \rightarrow 0}\left\langle\hbar^{-1 / 2} \alpha\left|q_{\hbar} \ldots p_{\hbar}\right| \hbar^{-1 / 2} \alpha\right\rangle=\xi \ldots \pi
$$

We shall show that (1.9) (in Weyl form) is preserved under the time evolution $U_{\hbar}(t)$ of any selfadjoint extension $H_{\hbar}$ of $p_{\hbar}^{2} / 2 m+V_{\hbar}$ :

$$
\lim _{\hbar \rightarrow 0}\left\langle\hbar^{-1 / 2} \alpha\left|q_{\hbar}(s) \ldots p_{\hbar}(t)\right| \hbar^{-1 / 2} \alpha\right\rangle=\xi(\alpha, s) \ldots \pi(\alpha, t),
$$


as long as the classical orbit exists. The fact, that along coherent states the quantum mechanical evolution $\left\langle\hbar^{-1 / 2} \alpha\left|a_{\hbar}(t)\right| \hbar^{-1 / 2} \alpha\right\rangle$ and the classical evolution $\xi(\alpha, t)=\left\langle\hbar^{-1 / 2} \alpha(t)\left|a_{\hbar}\right| \hbar^{-1 / 2} \alpha(t)\right\rangle$ are in "weak correspondence" (which becomes exact for $\hbar \rightarrow 0$ ) has been analyzed by Klauder [9]. But to the best of the present author's knowledge no general proof has been given of (1.10), nor has it been recognized that also (1.8) is preserved under time-evolution (for the technical details, see Theorem 2.1 and [37] for a probabilistic setting):

$$
\begin{gathered}
\lim _{\hbar \rightarrow 0}\left\langle\hbar^{-1 / 2} \alpha\left|\hbar^{-1 / 2}\left(q_{\hbar}(s)-\xi(\alpha, s)\right) \ldots \hbar^{-1 / 2}\left(p_{\hbar}(t)-\pi(\alpha, t)\right)\right| \hbar^{-1 / 2} \alpha\right\rangle \\
=\langle 0|q(\alpha, s) \ldots p(\alpha, t)| 0\rangle .
\end{gathered}
$$

Here the $q(\alpha, t)$ and $p(\alpha, t)$ are solutions of the linearized classical equations (1.2) around $\xi(\alpha, t)$ :

$$
\dot{q}(\alpha, t)=p(\alpha, t) / m, \quad \dot{p}(\alpha, t)=-\nabla V(\xi(\alpha, t)) q(\alpha, t),
$$

with initial conditions $q(\alpha, 0)=q, p(\alpha, 0)=p$. Both, (1.10) and (1.11) have an easy generalization to more complicated Hamiltonians and to relativistic and non-relativistic infinite boson systems. In the latter case the compensation of singularities for $\hbar \rightarrow 0$, when expanding the quantum dynamics around a classical solution, is implicit in the work of Goldstone [11] and Gross [12], but again a mathematical proof is desirable.

Our work has been most strongly influenced by the findings of Lieb and the author [13] in mean field models, as lasers and strongly coupled superconductors, that "intensive" quantities $a_{N}(t)$, i.e. space averages $N^{-1} \sum_{n=1}^{N} A_{n}$ of local observables translated in time by mean field Hamiltonians, become classical $\alpha(t)$ in the limit $N \rightarrow \infty$ along classical states, while the "fluctuations" $\sqrt{N}\left(a_{N}(t)-\alpha(t)\right)$ become boson operators $a(\alpha, t)$, which follow linearized equations of motion, if in the classical states the fluctuations at $t=0$ have a limit. We think that the analogy between $N \rightarrow \infty$ and $\hbar \rightarrow 0$ is significant for the understanding of classical operations within the framework of quantum mechanics [14]. It is the pedagogical goal of this paper to elaborate a unified picture of the classical limit in quantum mechanical correlation functions, which is so simple that it could belong into an elementary course on quantum mechanics.

The author is indebted to M. Fierz, J. Glimm, A. M. Jaffe, J. R. Klauder, B. Kostant and J. Lascoux for helpful discussions and bibliographical information and, last but not least, to E. H. Lieb whithout whom this paper would never have been written. 


\section{§ 2. Finitely Many Degrees of Freedom}

The passage to the classical limit in quantum mechanical correlation functions can be completely illustrated for the Hamiltonian $\mathscr{H}(\pi, \xi)$ $=\pi^{2} / 2 m+V(\xi)$ with only one degree of freedom:

Theorem 2.1. Let $V(\xi)$ be real and $\xi(\alpha, t)$ a solution of (1.2) for $|t|<T>0$ and initial data $\alpha$. Let $V$ be $C^{2+\delta}, \delta>0$, in a neighborhood of $\xi(\alpha, t)$ and assume that $\int|V(x)|^{2} \exp \left(-\varrho x^{2}\right) d x<\infty$ for some $\varrho<\infty$. Let $H_{\hbar}$ be any selfadjoint extension of

$$
-\frac{\hbar}{2 m} d^{2} / d x^{2}+V(\sqrt{\hbar} x) \text { in } L^{2}\left(\mathbb{R}^{1}\right) \quad \text { and } \quad U_{\hbar}(t)=\exp -i H_{\hbar} t / \hbar .
$$

Then for all $(r, s) \in \mathbb{R}^{2}$ and uniformly on compacts in $\{|t|<T\}$ :

$$
\begin{aligned}
& \operatorname{s-lim}_{\hbar \rightarrow 0} U\left(\hbar^{-1 / 2} \alpha\right)^{*} U_{\hbar}(t)^{*} \exp i\left[r\left(q-\hbar^{-1 / 2} \xi(\alpha, t)\right)+s\left(p-\hbar^{-1 / 2} \pi(\alpha, t)\right)\right] \\
& \text { and } \begin{aligned}
\cdot U_{\hbar}(t) U\left(\hbar^{-1 / 2} \alpha\right) & =\exp i[r q(\alpha, t)+s p(\alpha, t)] \\
s-\lim _{\hbar \rightarrow 0} U\left(\hbar^{-1 / 2} \alpha\right)^{*} & U_{\hbar}(t)^{*} \exp i\left[r q_{\hbar}+s p_{\hbar}\right] U_{\hbar}(t) U\left(\hbar^{-1 / 2} \alpha\right) \\
= & \exp i[r \xi(\alpha, t)+s \pi(\alpha, t)] .
\end{aligned}
\end{aligned}
$$

Here $(p(\alpha, t), q(\alpha, t))$ are the solutions of (1.2) linearized around $\xi(\alpha, t)$ with initial data $(p, q)$, which arise from the selfadjoint Hamiltonian

$$
H(t)=p^{2} / 2 m+V^{\prime \prime}(\xi(\alpha, t)) q^{2} / 2 .
$$

Proof. One expands $H_{\hbar} / \hbar$ around the classical orbit $\xi(\alpha, t)=\xi_{t}$ :

$$
\begin{aligned}
H_{\hbar} / \hbar & =H_{\hbar}^{0}(t)+H_{\hbar}^{1}(t)+H_{\hbar}^{2}(t)+H_{\hbar}^{3}(t), \\
H_{\hbar}^{0}(t) & =\mathscr{H}(\pi, \xi) / \hbar \\
H_{\hbar}^{1}(t) & =\pi_{t}\left(p-\hbar^{-1 / 2} \pi_{t}\right) \hbar^{-1 / 2}+V^{\prime}\left(\xi_{t}\right)\left(q-\hbar^{-1 / 2} \xi_{t}\right) \hbar^{-1 / 2}, \\
H_{\hbar}^{2}(t) & =\left(p-\hbar^{-1 / 2} \pi_{t}\right)^{2} / 2+V^{\prime \prime}\left(\xi_{t}\right)\left(q-\hbar^{-1 / 2} \xi_{t}\right)^{2} / 2 .
\end{aligned}
$$

The propagator $U_{\hbar}^{1}(t)=T \exp -i \int_{0}^{t} d s H_{h}^{1}(s)$ exists for all $|t|<T$ (by the unitary extension of its strongly convergent Dyson series on the linear hull of all Hermite functions) and defines an automorphism of the Weyl algebra:

$$
U_{\hbar}^{1}(t)^{*}\left(a^{\#}-\hbar^{-1 / 2} \alpha_{t}^{\#}\right) U_{\hbar}^{1}(t)=a^{\#}-\hbar^{-1 / 2} \alpha^{\#} .
$$

Hence the 1.h.s. of (2.1) can be written as

$$
W_{\hbar}(t, 0)^{*} \exp i[r q+s p] W_{\hbar}(t, 0),
$$


where

$$
\begin{aligned}
W_{\hbar}(t, s)= & U\left(\hbar^{-1 / 2} \alpha\right)^{*} U_{\hbar}^{1}(t)^{*} U_{\hbar}(t-s) U_{\hbar}^{1}(s) U\left(\hbar^{-1 / 2} \alpha\right) \\
& \cdot \exp i \int_{s}^{t} d r H_{\hbar}^{0}(r) .
\end{aligned}
$$

Hence (2.1) is proved, if on a dense subspace $s$ - $\lim W_{\hbar}(t, s)=W(t, s)$ $=T \exp -\int_{s}^{t} d r H(r)$ holds.

The normalized states $\left\{\psi_{a}(x)=\pi^{-1 / 4} \exp -(x-a)^{2} / 2 \mid a \in \mathbb{R}\right\}$ span $L^{2}(\mathbb{R})$. We claim that for every $0<k<T$ there exists some $\hbar_{k}>0$, such that for all $\hbar<\hbar_{k}$ and all $|s| \leqq k$, the total set of states

$\left\{\psi_{a}^{\hbar s}=U_{\hbar}^{1}(s) U\left(\hbar^{-1 / 2} \alpha\right) W(s, 0) \psi_{a}\right\} \subset D\left(p^{2}\right) \cap D\left(\hbar^{-1} V(\sqrt{\hbar} q)\right)$.

For, $H(r)$ is quadratic with $V^{\prime \prime}\left(\xi_{r}\right)$ continuous in $r$. Hence the Dyson series for $W(t, s)$ converges for small $|t-s|$ and

$$
\begin{aligned}
W(s, 0) q W(s, 0)^{*} & =\alpha q+\beta p, \\
W(s, 0) p W(s, 0)^{*} & =\gamma q+\delta p, \\
A=A(s) & =\left(\begin{array}{ll}
\alpha & \beta \\
\gamma & \delta
\end{array}\right) \in \operatorname{Sp}(2, \mathbb{R}),
\end{aligned}
$$

with continuous dependence on $s$. Since $\psi_{a}$ satisfies $[q-a+i p] \psi_{a}=0$,

or

$$
\begin{aligned}
0 & =U_{\hbar}^{1}(s) U\left(\hbar^{-1 / 2} \alpha\right) W(s, 0)[q-a+i p] \psi_{a} \\
& =\left[(\alpha+i \gamma)\left(q-\hbar^{-1 / 2} \xi_{s}\right)-a+i(\delta-i \beta)\left(p-\hbar^{-1 / 2} \pi_{s}\right)\right] \psi_{a}^{\hbar s},
\end{aligned}
$$

$\psi_{a}^{\hbar s}(x)=$ const $\exp \left[-\frac{(\alpha+i \gamma)}{2(\delta-i \beta)}\left(x-\hbar^{-1 / 2} \xi_{s}-\frac{a}{(\alpha+i \gamma)}\right)^{2}+i \pi_{s} \hbar^{-1 / 2} x\right]$.

Since $\operatorname{Re}(\alpha+i \gamma) /(\delta-i \beta) 2=1 / 2\left(\delta^{2}+\beta^{2}\right)>\eta_{k}>0$ for all $|s| \leqq k$, and since $\int d x|V(x)|^{2} \exp -\varrho x^{2}<\infty$ for some $\varrho<\infty$, one obtains (2.11) for $\hbar_{k}=2 \eta_{k} / \varrho$.

Therefore $W_{\hbar}(t, s) W(s, r) \psi_{a}$ is strongly differentiable with respect to $s$, if $0<k<T,|s|,|t| \leqq k$ and if $\hbar<\hbar_{k}$, for any selfadjoint extension $H_{\hbar}$ of $\mathrm{p}_{\hbar}^{2} / 2 m+V_{\hbar}$. We obtain the Duhamel formula

$$
\begin{aligned}
& W(t, 0) \psi_{a}-W_{\hbar}(t, 0) \psi_{a}=\int_{0}^{t} d s \frac{d}{d s} W_{\hbar}(t, s) W(s, 0) \psi_{a}, \\
& \frac{d}{d s} W_{\hbar}(t, s) W(s, 0) \psi_{a}=i W_{\hbar}(t, s)\left\{\hbar^{-1} V\left(\xi_{s}+\sqrt{\hbar} q\right)\right. \\
& \left.-\hbar^{-1} V\left(\xi_{s}\right)-\hbar^{-1 / 2} V^{\prime}\left(\xi_{s}\right) q-V^{\prime \prime}\left(\xi_{s}\right) q^{2} / 2\right\} W(s, 0) \psi_{a} .
\end{aligned}
$$


The norm of (2.16) will be estimated as follows: There exists some $\sigma>0$, such that $V\left(\xi_{s}+x\right)$ is $C^{2+\delta}$ for all $|s| \leqq k$ and $|x| \leqq \sigma$. We consider

$$
\begin{gathered}
\int d x\left|\hbar^{-1} V\left(\xi_{s}+\sqrt{\hbar} x\right)-\hbar^{-1} V\left(\xi_{s}\right)-\hbar^{-1 / 2} V^{\prime}\left(\xi_{s}\right) x-V^{\prime \prime}\left(\xi_{s}\right) x^{2} / 2\right|^{2} \\
\cdot\left|\left(W(s, 0) \psi_{a}\right)(x)\right|^{2} .
\end{gathered}
$$

In $\left\{|x| \geqq \hbar^{-1 / 2} \sigma\right\}$, each term is $O\left(\hbar^{N}\right)$ for every $N$, since $\left|V\left(\xi_{s}+\sqrt{\hbar} x\right)\right|^{2}$ increases at infinity at most as exp $\hbar \varrho x^{2}$, while $\left|\left(W(s, 0) \psi_{a}\right)(x)\right|^{2}$ decreases as $\exp -2 \eta_{k} x^{2}$. On the other hand, for $|x| \leqq \hbar^{-1 / 2} \sigma$, one uses the Hölder continuity of $V^{\prime \prime}(\delta \leqq 1)$ :

$$
\begin{aligned}
& \left|\hbar^{-1} V\left(\xi_{s}+\sqrt{\hbar} x\right)-\hbar^{-1} V\left(\xi_{s}\right)-\hbar^{-1 / 2} V^{\prime}\left(\xi_{s}\right) x-V^{\prime \prime}\left(\xi_{s}\right) x^{2} / 2\right| \\
& \leqq x^{2} \int_{0}^{1} d y(1-y)\left|V^{\prime \prime}\left(\xi_{s}+\sqrt{\hbar} x y\right)-V^{\prime \prime}\left(\xi_{s}\right)\right| \leqq \operatorname{const} x^{2+\delta} \hbar^{\delta / 2} .
\end{aligned}
$$

Hence $\left\|W(t, 0) \psi_{a}-W_{h}(t, 0) \psi_{a}\right\|=O\left(\hbar^{\delta / 2}\right)$ leads to (2.1). By the same argument

$$
\begin{gathered}
\left\|U\left(\hbar^{-1 / 2} \alpha\right)^{*} U_{\hbar}(t)^{*} e^{i\left[r q_{\hbar}+s p_{\hbar}\right]} U_{\hbar}(t) U\left(\hbar^{-1 / 2} \alpha\right) \psi-e^{i\left[r \xi_{t}+s \pi_{t}\right]} \psi\right\| \\
=\left\|W_{\hbar}(t, 0)^{*} \exp i \sqrt{\hbar}(r q+s p) W_{\hbar}(t, 0) \psi-\psi\right\| .
\end{gathered}
$$

Since $s-\lim W_{\hbar}(t, 0)=W(t, 0)$ and $s-\lim \exp i \sqrt{\hbar}(r q+s p)=\mathbb{1},(2.2)$ follows

Q.E.D.

Remark. It is helpful for the interpretation of Theorem 2.1 to note the analogy to time dependent scattering theory [15] between

$$
\lim _{\hbar \rightarrow 0}\left\|U_{\hbar}(t) U\left(\hbar^{-1 / 2} \alpha\right) \psi-U\left(\hbar^{-1 / 2} \alpha_{t}\right) W(t, 0) \psi\right\|=0
$$

and

$$
\lim _{t \rightarrow \infty}\left\|e^{i H t} \Omega_{-} \psi-e^{i H_{0} t} \psi\right\|=0 .
$$

If $\psi$ is Gaussian, then also $U\left(\hbar^{-1 / 2} \alpha\right) \psi$ and $U\left(\hbar^{-1 / 2} \alpha_{t}\right) W(t, 0) \psi$, and (2.20) shows that under the time evolution $U_{\hbar}(t)$ the difference of $U_{\hbar}(t) U\left(\hbar^{-1 / 2} \alpha\right) \psi$ from a Gaussian wave packet centered around the classical orbit and with the shape wobbling according to the quadratic Hamiltonian of the linearized theory goes to zero, as $\hbar \rightarrow 0$.

The error in (2.2) between Gaussian wave packets can be reduced to $O(\sqrt{\hbar})$ uniformly for bounded time intervals, if $\xi(\alpha, t)$ exists for all $t$ and if $V$ is $C^{3}$ in a neighborhood of this orbit. Hence the Ehrenfest theorem describes well the classical aspects of the motion of wave packets for finite times, but not for $t \rightarrow \infty$. Our method is complementary to the WKB-method, which is successful for describing the stationary states in quantum mechanics.

One learns from Theorem 2.1 that equilibrium points $\left(\pi_{0}, \xi_{0}\right)$ of the classical motion, $\pi_{0}=0$ and $V^{\prime}\left(\xi_{0}\right)=0$, are driven by the quantum 
fluctuations in $O(\sqrt{\hbar})$ : If $V^{\prime \prime}\left(\xi_{0}\right)>0$, then (2.3) leads to an oscillatory behavior of $\Delta p^{2}$ and $\Delta q^{2}$ in any wave packet $\psi_{t}$. For $V^{\prime \prime}\left(\xi_{0}\right) \leqq 0$ the spectrum of (2.3) is purely continuous and the wave packets spread, for $V^{\prime \prime}\left(\xi_{0}\right)=0$ with a power law and exponentially fast for $V^{\prime \prime}\left(\xi_{0}\right)<0$.

A slight modification of the kinematics leads to the classical limit for heavy particles, if $\lambda=\hbar / m \rightarrow 0$ in Hamiltonians

$$
\mathscr{H}(\pi, \xi)=\pi^{2} / 2 m+m V(\xi) .
$$

Corollary 2.2. Consider (2.22) (under the same assumptions on $V$ as in Theorem 2.1) around the solution $\xi(t)$ of the classical equation $\ddot{\xi}(t)=-V^{\prime}(\xi(t))$ with initial data $\alpha=(\xi(0)+i \dot{\xi}(0)) / \sqrt{2}$. Let

$$
p_{\lambda}=m \sqrt{\lambda} p, \quad q_{\lambda}=\sqrt{\lambda} q,
$$

and let $H_{\lambda} \hbar^{-1}$ be any selfadjoint extension of $p^{2} / 2+\lambda^{-1} V(\sqrt{\lambda} q)$ with $U_{\lambda}(t)=\exp -i H_{\lambda} t / \hbar$. Then

$$
\begin{aligned}
& s-\lim _{\lambda \rightarrow 0} U^{*}\left(\lambda^{-1 / 2} \alpha\right) U_{\lambda}(t)^{*} \exp i\left[r\left(q-\lambda^{-1 / 2} \xi_{t}\right)+s\left(p-\lambda^{-1 / 2} \dot{\xi}_{t}\right)\right] \\
& \text { - } U_{\lambda}(t) U\left(\lambda^{-1 / 2} \alpha\right)=\exp i[r q(t)+s p(t)], \\
& s-\lim _{\lambda \rightarrow 0} U\left(\lambda^{-1 / 2} \alpha\right)^{*} U_{\lambda}(t)^{*} \exp i\left[r q_{\lambda}+s p_{\lambda} / m\right] U_{\lambda}(t) U\left(\lambda^{-1 / 2} \alpha\right) \\
& =\exp i\left[r \xi_{t}+s \dot{\xi}_{t}\right] \text {, }
\end{aligned}
$$

where $\dot{q}(t)=p(t), \dot{p}(t)=-V^{\prime \prime}\left(\xi_{t}\right) q(t), q(0)=q, p(0)=p$.

For $N$-particle Hamiltonians of the type

$$
\mathscr{H}(\pi, \xi)=\sum_{n=1}^{N}\left(\pi_{n}-\frac{e}{c} \boldsymbol{A}\left(\xi_{n}, t\right)\right)^{2} / 2 M_{n}+V(\xi, t)
$$

with nontrivial time-dependence, we have to assume the following regularity property:

$(R)$ : There exists a propagator $U_{\hbar}(t, s)$ which is strongly continuous for $-\infty<s, t<+\infty$ with $U_{h}(t, s) U_{\hbar}(s, r)=U_{\hbar}(t, r), U_{\hbar}(t, s)^{*}=U_{\hbar}(s, t)$ and $U_{\hbar}(t, t)=\mathbb{1}$. For some $\varrho<\infty$ and all Gauss packets with

$$
\begin{gathered}
\sup \left|\psi(x) \exp \varrho\|x\|^{2}\right|<\infty, \\
s-\lim _{r \rightarrow 0} \hbar r^{-1}\left[U_{\hbar}(t, s+r)-U_{\hbar}(t, s)\right] \psi=i U_{\hbar}(t, s) H_{\hbar}(s) \psi,
\end{gathered}
$$

where $H_{\hbar}(s) \psi$ is naturally defined as partial differential operator.

Theorem 2.3. For $N$-body systems (2.26) with $\boldsymbol{A}$ and $V$ satisfying $(R)$, a generalized Ehrenfest theorem of the type (2.1), (2.2) holds along every classical orbit $\xi(t)$, in the neighborhood of which $A$ and $V$ are $C^{2+\delta}, \delta>0$. 
In the classical limit, there is no difference between the coherent and incoherent superposition of states of the type $U\left(\hbar^{-1 / 2} \alpha_{n}\right) \varphi_{n}$ [with $\alpha_{n} \in \mathbb{C}^{f}$ and $\left.\varphi_{n} \in L^{2}\left(\mathbb{R}^{f}\right)\right]$, if $\Sigma\left\|\varphi_{n}\right\|^{2}=1$ and $\alpha_{m} \neq \alpha_{n}$ for $m \neq n$ : For all $(r, s) \in \mathbb{R}^{2}$ and $|t|<\min T\left(\alpha_{n}\right)$ and

$$
\begin{gathered}
\psi_{\hbar}=\Sigma U\left(\hbar^{-1 / 2} \alpha_{n}\right) \varphi_{n}, \quad P_{\hbar}=\Sigma U\left(\hbar^{-1 / 2} \alpha_{n}\right)\left|\varphi_{n}\right\rangle\left\langle\varphi_{n}\right| U\left(\hbar^{-1 / 2} \alpha_{n}\right)^{*}, \\
\lim _{\hbar \rightarrow 0}\left(\psi_{\hbar}, U_{\hbar}(t)^{*} e^{i\left[r q_{\hbar}+s p_{\hbar}\right]} U_{\hbar}(t) \psi_{\hbar}\right) \\
=\lim _{\hbar \rightarrow 0} \operatorname{Tr}\left(P_{\hbar} U_{\hbar}(t)^{*} e^{i\left[r q_{\hbar}+s p_{\hbar}\right]} U_{\hbar}(t)\right) \\
=\sum_{n}\left\|\varphi_{n}\right\|^{2} \exp i\left[r \xi\left(\alpha_{n}, t\right)+s \pi\left(\alpha_{n}, t\right)\right] .
\end{gathered}
$$

This is important for fermions or bosons, where the (anti-)symmetrization of spatial wave functions of the type $U\left(\hbar^{-1 / 2} \alpha\right) \varphi$ usually leads to a classical ensemble in phase space with discrete density. Classical ensembles with continuous densities $\varrho(\pi, \xi) \geqq 0, \int \mathrm{d} \pi \mathrm{d} \xi \varrho(\pi, \xi)=1$ can obviously be reached from any density matrix $P$, by forming

$$
P_{\hbar}=\int d \pi d \xi \varrho(\pi, \xi) U\left(\hbar^{-1 / 2} \alpha\right) P U\left(\hbar^{-1 / 2} \alpha\right)^{*}
$$

and by passing to the limit as in (2.27) for $|t|<\min T(\alpha)$.

A classical problem is the limit $\hbar \rightarrow 0$ and the related high temperature expansion in statistical mechanics $[16,17]$ :

$$
\begin{aligned}
& \lim _{\hbar \rightarrow 0} h^{f} \operatorname{Tr} e^{-\beta H_{\hbar}}=\int d \pi d \xi e^{-\beta \mathscr{H}(\pi, \xi)}, \\
& \lim _{\hbar \rightarrow 0} \operatorname{Tr}\left(e^{-\beta H_{\hbar}} p_{\hbar}^{m} q_{\hbar}^{n}\right) / \operatorname{Tr} e^{-\beta H_{\hbar}} \\
& =\int d \pi d \xi e^{-\beta \mathscr{H}(\pi, \xi)} \pi^{m} \xi^{n} / \int d \pi d \xi e^{-\beta \mathscr{H}(\pi, \xi)} .
\end{aligned}
$$

For finite $f,(2.29)$ has been proved by Berezin [18] for a large class of Hamiltonians. In [19] the limit (2.30) of the correlation functions was considered for finitely many harmonic oscillators compled linearly to large systems of multilevel atoms. This method can be generalized to $N$-particle systems in an anharmonic oszillator well and interacting via regular short range two-body potentials.

Finally let us remark that there exist coherent states on a large class of Lie groups [20], which allow the passage to the classical limit for dynamical systems with more exotic phase spaces than $\mathbb{R}^{2 f}[21-23]$. One example, $\mathbb{R}^{2} \times \mathrm{SU}_{2}$, with "atomic" coherent states $[24,25]$ for $\mathrm{SU}_{2}$, is important in the thermodynamic limit of the laser [13]. 


\section{§ 3. Boson Systems of Infinitely Many Degrees of Freedom}

Some of the results of the preceeding section can be generalized to systems with infinitely many degrees of freedom.

The best understood models for a relativistic quantum dynamics are the scalar boson theories in two dimensional space time with polynomial interaction (see e.g. [26]). Let $\Phi(x), x \in \mathbb{R}^{1}$, be the free boson field of mass $m>0$ at $t=0, H_{0}\left(m^{2}\right)$ the corresponding free Hamiltonian and $\Pi(x)=i\left[H_{0}, \Phi(x)\right]$ in Fock space $\mathscr{F}$ with $[\Phi(x), \Phi(y)]=[\Pi(x), \Pi(y)]=0$ and $[\Phi(x), \Pi(y)]=i \delta(x-y)$. Let $\Phi(f)=\int d x f(x) \Phi(x)$ for $f \in \mathscr{D}\left(\mathbb{R}^{1}\right)$. For every $\alpha \in \mathscr{D}\left(\mathbb{R}^{1}\right)$ with the decomposition $\alpha(x)=(\varphi(x)+i \pi(x)) / \sqrt{2}$ into real and imaginary part, the shift operator $U(\alpha)$ satisfies

$$
\begin{aligned}
U(\alpha)= & \exp i[\Phi(\pi)-\Pi(\varphi)] \\
U(\alpha)^{*}: \Phi(x)^{m}: U(\alpha)= & :(\Phi(x)+\varphi(x))^{m}: \\
U(\alpha)^{*}: \Pi(x)^{m}: U(\alpha)= & :(\Pi(x)+\pi(x))^{m}: \\
U(\alpha)^{*} H_{0} U(\alpha)= & H_{0}+\int d x\{\pi(x) \Pi(x)+\nabla \varphi \nabla \Phi(x) \\
& \left.+m^{2} \varphi(x) \Phi(x)\right\}+\mathscr{H}_{0}(\alpha)
\end{aligned}
$$

where $\mathscr{H}_{0}(\alpha)$ is the classical energy of the free field $\left(\square+m^{2}\right) \varphi(x, t)=0$ with Cauchy data $\alpha$, and : : is the Wick ordering w.r.t. the free vacuum.

For Cauchy data $\alpha \in \mathscr{D}\left(\mathbb{R}^{1}\right)$, the classical nonlinear real wave equation

$$
\left(\square+m^{2}\right) \varphi(\alpha, t, x)+\sum_{n=1}^{N} n a_{n} \varphi(\alpha, t, x)^{n-1}=0
$$

has for finite times, $|t|<T(\alpha)>0$, a unique smooth solution with propagation speed 1 (see [27-29]), where $T(\alpha)=\infty$ for $N$ even and $a_{N}>0$. These solutions will be compared with the quantum solutions of

$$
\left(\square+m^{2}\right) \Phi_{h}(t, x)+\sum_{n=1}^{N} n a_{n}: \Phi_{h}(t, x)^{n-1}:
$$

with $\Phi_{\hbar}(0, x)=\Phi_{\hbar}(x)=\sqrt{\hbar} \Phi(x), \Pi_{\hbar}(0, x)=\Pi_{\hbar}(x)=\sqrt{\hbar} \Pi(x)$, which have been constructed by Glimm and Jaffe [26] for $N$ even and $a_{N}>0$.

Let $r>0$ and $0 \leqq g_{r} \in \mathscr{D}\left(\mathbb{R}^{1}\right)$ with $g_{r}(x)=1$ for $|x| \leqq r$. Let $H_{h r}$ be any self-adjoint extension of $\hbar H_{0}+V_{\hbar}\left(g_{r}\right)$ from $D\left(H_{0}\right) \cap D\left(V_{\hbar}\left(g_{r}\right)\right)$, where

$$
V_{\hbar}\left(g_{r}\right)=\sum_{n=1}^{N} a_{n} \int d x g_{r}(x): \Phi_{\hbar}^{n}(x): .
$$

Let $U_{\hbar r}(t)=\exp -i t H_{\hbar r} / \hbar$. On $D\left(H_{0}\right) \cap D\left(V_{\hbar}\left(g_{r}\right)\right)$ the generator of $U_{\hbar r}(t)$ is

$$
H_{0}+\sum_{n=1}^{N} a_{n} \hbar^{\frac{n}{2}-1} \int d x g_{r}(x): \Phi(x)^{n}: \text {. }
$$


Theorem 3.1. Let $\alpha \in \mathscr{D}\left(\mathbb{R}^{1}\right)$ and $\varphi(\alpha, t, x)$ exist for $|t|<T$. Let $f, g \in \mathscr{D}\left(\mathbb{R}^{1}\right)$ be real and $r$ be sufficiently large. Then

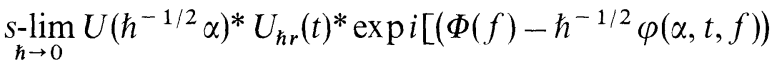

$$
\begin{aligned}
& \left.+\left(\Pi(g)-\hbar^{-1 / 2} \pi(\alpha, t, g)\right)\right] U_{\hbar r}(t) U\left(\hbar^{-1 / 2} \alpha\right) \\
& =\exp i[\Phi(\alpha, t, f)+\Pi(\alpha, t, g)] \text {, } \\
& \underset{\hbar-\lim }{\hbar \rightarrow 0} U\left(\hbar^{-1 / 2} \alpha\right)^{*} U_{\hbar r}(t)^{*} \exp i\left[\Phi_{\hbar}(f)+\Pi_{\hbar}(g)\right] U_{\hbar r}(t) U\left(\hbar^{-1 / 2} \alpha\right) \\
& =\exp i[\varphi(\alpha, t, f)+\pi(\alpha, t, g)] \text {, }
\end{aligned}
$$

where $\varphi(\alpha, t, f)=\int d x f(x) \varphi(\alpha, t, x), \Phi(\alpha, t, f)=\int d x f(x) \Phi(\alpha, t, x)$, and where the $\Phi(\alpha, t, x)$ and $\Pi(\alpha, t, x)=\dot{\Phi}(\alpha, t, x)$ are the unique global solutions of (3.3) linearized around $\varphi(\alpha, t, x)$ :

$$
0=\left(\square+m^{2}\right) \Phi(\alpha, t, x)+\sum_{n=2}^{N} n(n-1) a_{n} \varphi(\alpha, t, x)^{n-2} \Phi(\alpha, t, x)
$$

with initial conditions $\Phi(x), \Pi(x)$ at $t=0$.

Proof. The proof of Theorem 2.1 applies with few changes. (3.6) is developed around $\varphi(\alpha, t, x)$ in an obvious way. Again

$$
H_{r}(t)=H_{0}+\sum_{n=2}^{N}\left(\begin{array}{l}
n \\
2
\end{array}\right) a_{n} \int d x g_{r}(x) \varphi(\alpha, t, x)^{n-2}: \Phi(x)^{2}:
$$

has a propagator $W_{r}(t, s)$, which generates (3.9) for $r$ sufficiently large. $W_{r}(t, s)$ has for small $|t-s|$ a controllable action on $\mathscr{F}_{0}$, the subspace of finite particle states with momentum space wave functions of compact support, by its convergent Dyson expansion. Here one easily sees that $s$-lim $W_{\hbar r}(t, s) \psi=W_{r}(t, s) \psi$ for $\hbar \rightarrow 0,|t-s|$ small, using the Duhamel formula. Hence, using unitarity and the composition law,

$$
W_{r}(t, u) W_{r}(u, s)=W_{r}(t, s), \quad s-\lim W_{\hbar r}(t, s)=W_{r}(t, s)
$$

for all $|s|,|t|<T$. Q.E.D.

Remark that by the Wick reordering automorphism one can transfer mass from $H_{0}$ to $V$. In the translation to the classical limit the coefficients in (3.6) are $\hbar$-dependent and make that only the unique highest order term in the transition from $\Phi\left(x_{1}\right) \ldots \Phi\left(x_{n}\right)$ to a Wick product $: \Phi(x)^{n}$ : contributes in the limit $\hbar \rightarrow 0$.

The classical limit in Theorem 3.1, which in perturbation theory corresponds to the sum over all tree graphs (see e.g. [30]), gives a rigorous meaning to the Goldstone picture [11] as the leading asymptotic term in an expansion in $\sqrt{\hbar}$. The $O(\sqrt{\hbar})$-correction gives an interesting instability, whenever the classical field equations have a non-zero 
stationary solution $\varphi_{0}$, e.g. for $2 a_{2}<-m^{2}, a_{4}>0$ and $a_{n}=0$ for $n \neq 2,4$. In this case, (3.10) with $\alpha(x)=\varphi_{0} / \sqrt{2}= \pm\left(-2 a_{2}-m^{2}\right)^{1 / 2}\left(24 a_{4}\right)^{-1 / 2}$ for $|x| \leqq r$ is locally equivalent to $H_{0}\left(-4 a_{2}-2 m^{2}\right)$ with positive mass elementary excitations, while for the unstable stationary state $\varphi(x, t) \equiv 0$ one has local equivalence to $H_{0}\left(m-2 a_{2}\right)$ with purely imaginary mass.

In more than 2-dimensional space-time, the renormalized local Hamiltonians for $\Phi_{3}^{4}$ [31] and $\Phi_{4}^{3}$ [32] are for $\hbar>0$ defined in non-Fock representations of the $\mathrm{CCR}$. Perturbation theory indicates that the classical limit is again of the structure of Theorem 3.1. In $\Phi_{4}^{4}$ one can introduce an ultraviolet cut-off at $|\boldsymbol{k}| \leqq \kappa=$ const $\hbar^{-1 / 3}$ and obtain the classical limit without any renormalization.

In non-relativistic many-body theory, the classical limit for bosons with the second quantized Hamiltonian (in Fock space over $L^{2}\left(\mathbb{R}^{3}\right)$ )

$$
\begin{gathered}
H_{\hbar}=-\frac{\hbar^{2}}{2 m} \int d x a^{*}(x) \Delta a(x) \\
\quad+\frac{1}{2} \int d x d y a^{*}(x) a^{*}(y) V(x-y) a(x) a(y) \\
{[a(x), a(y)]=0, \quad\left[a(x), a^{*}(y)\right]=\delta(x-y),}
\end{gathered}
$$

has been discussed by Gross [12] as the first step in a series of canonical transformations for diagonalizing $H_{\hbar}$ in the thermodynamic limit.

We shall assume $V(x)=V(-x)=V(x)^{*}$ to be a Kato potential [15] and $\partial V / \partial x_{i}, \partial^{2} V / \partial x_{i} \partial x_{j}$ to be $-\Delta$-bounded. By a fixed-point argument one can show that for every initial condition $\beta \in D(\Delta)$ there exists a unique solution of the classical non-linear wave equation

$\frac{\partial \alpha}{\partial t}(\beta, t, x)=\frac{i}{2 \mu} \Delta \alpha(\beta, t, x)+i \int d y V(x-y)|\alpha(\beta, t, y)|^{2} \alpha(\beta, t, x)$,

with $\alpha(\beta, t, \cdot) \in D(\Delta)$ for $|t|<T>0$ and $\alpha(\beta, 0, \cdot)=\beta$. Furthermore, $H_{\hbar}$ is essentially self-adjoint on $\mathscr{F}_{0}$. Let $\beta \in D(\Delta), a^{\#}(\beta)=\int d x \beta(x) a^{\#}(x)$ and $U(\beta)=\exp \left[a^{*}(\beta)-a\left(\beta^{*}\right)\right]$. In an almost coherent state $U\left(\hbar^{-1 / 2} \beta\right) \varphi$, $\varphi \in \mathscr{F}_{0}$, the particle number is $O\left(\hbar^{-1}\right)$ for $\hbar \rightarrow 0$. However, $\hbar^{-1} H_{\hbar}$ is not extensive, as in Theorem 3.1, since $\hbar^{-1} H_{0 \hbar}=O(1)$ and $\hbar^{-1} V_{\hbar}=O\left(\hbar^{-3}\right)$ for $\hbar \rightarrow 0$. A non-trivial classical limit can be obtained by setting $m=\hbar^{3} \mu$, $t=\hbar^{2} \tau$ and by keeping $\mu>0$ and $\tau$ fixed. This leads to $U_{\hbar}(\tau)=\exp -i K_{\hbar} \tau$ $=\exp -i H_{\hbar} t / \hbar$, where

$K_{\hbar}=-\frac{1}{2 \mu} \int d x a^{*}(x) \Delta a(x)+\frac{\hbar}{2} \int d x d y a^{*}(x) a^{*}(y) V(x-y) a(x) a(y)$.

For $\gamma \in L^{2}\left(\mathbb{R}^{3}\right)$ we set $\alpha^{\#}(\beta, t, \gamma)=\int d x \gamma(x) \alpha^{\#}(\beta, t, x)$ and $a^{\#}(\beta, t, \gamma)$ $=\int d x \gamma(x) a^{\#}(\beta, t, x)$, where $a(\beta, t, x)$ are the solutions of the linearization of (3.12) around $\alpha(t, \beta, x)$ with initial data $a(x)$. Furthermore, let $a_{\hbar}(x)=\sqrt{\hbar} a(x)$. Then 
Theorem 3.2. Under the above assumptions one has for $|\tau|<T$ :

$$
\begin{aligned}
s-\lim & U\left(\hbar^{-1 / 2} \beta\right)^{*} U_{\hbar}(\tau)^{*} \exp \left[\left(a^{*}(\gamma)-\hbar^{-1 / 2} \alpha^{*}(\beta, \tau, \gamma)\right)-\text { h.c. }\right] \\
& \cdot U_{\hbar}(\tau) U\left(\hbar^{-1 / 2} \beta\right) \\
= & \exp \left[a^{*}(\beta, \tau, \gamma)-a\left(\beta, \tau, \gamma^{*}\right)\right], \\
s-\lim _{\hbar \rightarrow 0} U\left(\hbar^{-1 / 2} \beta\right)^{*} U_{\hbar}(\tau)^{*} \exp \left[a_{\hbar}^{*}(\gamma)-a_{\hbar}\left(\gamma^{*}\right)\right] U_{\hbar}(\tau) U\left(\hbar^{-1 / 2} \beta\right) & \exp \left[\alpha^{*}(\beta, \tau, \gamma)-\alpha\left(\beta, \tau, \gamma^{*}\right)\right] .
\end{aligned}
$$

Proof. As in Theorem 3.1 one proves $s-\lim W_{\hbar}(t, s) \psi=W(t, s) \psi$ first for $\psi \in \mathscr{F}_{0}$, by using the Dyson series for $W(t, s)$ and the Duhamel formula for small $|t-s|$.

It is amusing but not surprising that the classical limit is not unique: in coherent states centered around $\hbar^{-1 / 2}\left(\alpha_{1}, \ldots, \alpha_{N}\right)$ with fixed $N$, one obtains the classical mechanics of $N$ mass points by Theorem 2.1, while in boson coherent states centered in Fock space around a classical field $\hbar^{-1 / 2} \alpha(x)$ one obtains a classical field theory, if $m \sim \hbar^{3}$ and $t \sim \hbar^{2}$.

The transition from the quantum to the classical correlation functions in Gibbs states in the thermodynamical limit is presently only understood for small activities, where the Kirkwood Salsburg equations have a unique solution (see [33-34], and [35] for the diagrammatic analysis).

\section{§4. Conclusion}

The main objective of this paper was to give a simple and mathematically rigorous discussion of the classical limit in quantum mechanics. We hope that our construction can sometimes be used as a reliable starting point for understanding some of the intriguing features of infinite quantum systems, as for the boson condensation and the appearance of broken symmetries.

\section{References}

1. Schrödinger, E.: Ann. d. Phys. 79, 489 (1926)

2. Heisenberg, W.: Die physikalischen Prinzipien der Quantentheorie. Leipzig: Hirzel 1930

3. Pauli,W.: Die allgemeinen Prinzipien der Wellenmechanik, Handbuch der Physik, V. 1, Berlin-Göttingen-Heidelberg: Springer 1958

4. Andrié, M.: Comment. Phys. Math. 41, 333 (1971)

5. Maslov, V.P.: Uspekhi Mat. Nauk, 15, 213 (1960); Théorie des perturbations et méthodes asymptotiques. Paris: Dunod 1972

6. Feynman, R.P.: Rev. Mod. Phys. 20, 367 (1948)

7. Nelson, E.: J. Math. Phys. 5, 332 (1964)

Berezin, F. A., Šubin, M. A.: Coll. Math. Soc. J. Bolyai, 5 (1970) 
8. Ehrenfest, P.: Z. Physik 45, 455 (1927)

9. Klauder, J. R.: J. Math. Phys. 4, 1058 (1963); 5, 177 (1964); 8, 2392 (1967)

10. Glauber, R. J.: Phys. Rev. 131, 2766 (1963)

11. Goldstone, J.: Nuovo Cim. 19, 154 (1961)

12. Gross, E.P.: Phys. Rev. 100, 1571 (1955); 106, 161 (1957); Ann. Phys. 4, 57 (1958); 9, 292 (1960)

13. Hepp,K., Lieb,E.H.: Ann. Phys. 76, 360 (1973); Helv. Phys. Acta 46 (1973). Constructive quantum field theory. Velo, G., Wightman, A.S., eds., Lecture Notes in Physics, Berlin-Heidelberg-New York: Springer 1973

14. Hepp, K.: Helv. Phys. Acta 45, 237 (1972)

15. Kato, T.: Perturbation theory of linear operators. Berlin-Heidelberg-New York: Springer 1966

16. Wigner, E.P.: Phys. Rev. 40, 749 (1932)

17. Kirkwood, J. G.: Phys. Rev. 44, 31 (1933); 45, 116 (1934)

18. Berezin,F.A.: Math. USSR Sbornik 15, 577 (1971); 17, 269 (1972); Izv. Akad. Nauk Ser. Mat. 37, 1134 (1972)

19. Hepp, K., Lieb, E. H.: Phys. Rev. A 8, 2517 (1973)

20. Perelomov, A.M.: Commun. math. Phys. 26, 222 (1972)

21. Kostant, B.: In: Group representations in mathematics and physics, Bargmann, V., ed., Berlin-Heidelberg-New York: Springer 1970

22. Souriau, J. M.: Structure des systèmes dynamiques. Paris: Dunod 1970

23. Kirillov, A.A.: Elements of representation theory. Moscow: Nauka 1972

24. Arecchi,F.T., Courtens, E., Gilmore, R., Thomas, H.: Phys. Rev. A 6, 2211 (1972)

25. Lieb, E. H.: Commun. math. Phys. 31, 327 (1973)

26. Glimm,J., Jaffe, A. M.: In: Mathematics of contemporary physics, Streater, R.F., ed., London: Academic P. 1972

27. Jörgens, K.: Math. Z. 77, 295 (1961)

28. Browder, F.E.: Math. Z. 80, 249 (1962)

29. Segal, I. E.: Ann. Math. 78, 339 (1963)

30. Coleman, S., Weinberg, E.: Phys. Rev. D 7, 1888 (1973)

31. Glimm, J.: Commun. math. Phys. 10, 1 (1968)

32. Osterwalder, K.: Fortschr. Physik 19, 43 (1971)

33. Ruelle,D.: Statistical mechanics: Rigorous results, New York: Benjamin 1969

34. Ginibre, J.: In: Statistical mechanics and quantum field theory, de Witt, C., Stora, R., eds. Paris: Gordon \& Breach 1971

35. Bloch, C., de Dominicis, C.: Nucl. Phys. 10, 181 (1959)

36. Bialynicki-Birula, I.: Ann. Phys. 67, 252 (1971)

37. Martin-Löf, A.: Skand. Aktuarietidskr. 1967, 70

Communicated by W. Hunziker

\author{
Klaus Hepp \\ Physics Department \\ E.T.H. \\ Hönggerberg \\ CH-8049 Zürich \\ Switzerland
}


